\title{
The Immunity of Random Boolean Networks Based on Importance Index of Nodes
}

\author{
Yunge Bai ${ }^{1, a}$, Binghui Guo*1,b ${ }^{1,}$ Jiabao Li $^{2}$, Wei Wei ${ }^{1}$, Zhiming Zheng ${ }^{1}$ \\ ${ }^{1}$ LMIB, BDBC and School of Mathematics and Systems Science, Beihang University, Beijing \\ 100191, China \\ ${ }^{2}$ LMIB and School of Mathematical Sciences, Peking University, Beijing 100871, China \\ aemail: baiyg@buaa.edu.cn, bemail: guobinghui@buaa.edu.cn(*corresponding author)
}

Keywords: Boolean network, Importance index, Immunity strategy.

Abstract. Random Boolean network is a model of gene regulation network developed by Kauffman in 1969.In this paper, we focus on the evolution of the configuration space of random Boolean network, by the perspective of nodes immune. To investigate the influence of different strategies with taking nodes by local centrality, we use the robustness as well as the average recovery step number as the index for the degree of immunity. The results of this investigation show that immune process will reduce the network robustness index, which is in disturbance will be back to the initial non-attracting sets of probability increases. Furthermore, the average recovery steps returned to steady speed increases at the same time. As contrast, the immunization based on importance effect of BA networks is more pronounced than random immunization.

\section{Introduction}

Boolean network was first proposed by Kauffman as a mathematical model of gene regulatory networks in 1969, also as known as N-K model or Kauffman network. [1,2]

In random Boolean networks, a total of $n$ genes as nodes, for each gene there are two states of expression and not expression can be represented as Boolean variables 0 and 1 that represent the expression of a gene promote or inhibit the other gene's expression. The relationship between this regulation is random Boolean network relations between nodes.

In the last decades of development, random Boolean have derived many different models depending on the application needs of a variety of different backgrounds and theory, it is widely used in science, mathematics, social studies and technology engineering life. Because of the random Boolean network modeling can be used to study these important areas, so depth study of the dynamic behavior of a random Boolean network, whether at the application level or in the theoretical level, have a very important significance. Various properties of Boolean networks have been research, such as activities and sensitivities. [3]

In this paper, we use the robustness [4] as well as the average recovery step number as the index for the effect of immunity of different random Boolean networks.. 


\section{Model and Methods}

We use Barabsi-Albert method [5,6] to generate random scale-free network. Then modify to its own directed graph get fit Boolean network rather than generated undirected graph. The map is combined with Boolean topology update function to get complete random Boolean networks.

In this model for each network, we set a fixed out-degree. The network is generated by a growth process. Each node added into network will choose nodes to connect by the BA method.

The Boolean network contains $\underline{n}$ nodes $\left\{x_{1}, x_{2}, \ldots, x_{n}\right\}$ and $\underline{n}$ Boolean functions $\left\{f_{1}, f_{2}, \ldots, f_{n}\right\}$.. Each node corresponds to a $k_{i}$ input node and its input function $f_{i}$. The update of the Boolean network is determined by the following equation

$$
x_{i}(t+1)=f_{i}\left(x_{1}(t), x_{2}(t), \ldots, x_{k_{i}}(t)\right)
$$

In this model, each node have the same out-degree. Each nodes state will influenced by the same number of nodes. For example, if the fixed out-degree is 3, each nodes have a 3-element function to decide nodes state. Than we randomly set that function to simulate more natural situations

The robustness index is used to reflect the ability of random Boolean networks to resist perturbation. For a attractor set $A$ of length $L$ of the given random Boolean network, the robustness $R_{A}$ is defined as the probability that the network state can return to the attracting set $\mathrm{A}$ after each disturbance of the $\mathrm{L}$ network states in A.

$$
R_{A}=C / N L
$$

There are $N$ components for each network state, there is a disturbance of the $N L$ kinds of distances 1 for the attracting set A of length L. For the state of this $N L$ disturbance, we start from it, and use the update function to evolve after the state of the disturbance, and evolve until the evolved state enters an attracting set. Set the total number of $A^{r}=A$ be C, $A^{\prime}$ is the attracting set, for each perturbation.

Let $A$ take all the attracting sets of a given random Boolean network, $\left\langle R_{A}\right\rangle$ is the average of all $R_{A}$, $\left\langle R_{A}\right\rangle$ is called the robustness index of stochastic Boolean networks, which reflects the ability of random Boolean network to resist perturbation. For a given parameter, we generate multiple random Boolean network instances and take the average of these $\left\langle R_{A}\right\rangle$, and the result is $\mathrm{R}$, which is the estimated value of the random Boolean network robustness index for this set of parameters.

The robustness exponent expresses the ability of the random Boolean network to resist the perturbation. And here set an index $R \#$ represents the average number of recovery steps to show the speed of recovery after the disturbance. The average number of recovery steps indicates the ability to return to a steady state after a network perturbation. The recovery in this metric does not specify whether to recover to the same attracting set before its disturbance.

The networks in the model in this paper are directed graphs, and all nodes have the same outdegree. The immunization strategy studied in this paper is based on the importance of nodes. In order to ensure the integrity of the experiment, and to facilitate the comparison of experimental results, so need to be immune and randomized immune results as a contrast, in order to reflect the advantages and disadvantages of the immune strategy.

\subsection{Immune strategy on network}

Definition of immunity in Boolean network: If a node is immune, it is not affected by the update function during the evolution of the network. That node's value is constant from the beginning to the end in the whole evolution process.

Definition of immunization based on degree [7]: For the graph $G=(V, E)$ the degree of nodes $i$ is defined as 


$$
k(i)=\sum_{j \in G} a_{i j}
$$

In this model, every node has the same out-degree. So, the ranking of the nodes' degree is the same as the sort of the in-degree. And choose the nodes that have top rank to immune.

Definition of immunization based on local centrality [7]: Local centrality is defined as

$$
L(i)=\sum_{j \in \Gamma(i)} \sum_{u \in \Gamma(j)} N(u)
$$

$\Gamma(i)$ represents the nearest neighbor set of node I, $N(u)$ represents the sum of the number of the nearest neighbor and second nearest neighbors of node $u$.

We compute a lot of random Boolean network example's robustness index to get a representative value of network stability.

We randomly select 10000 initial states for each network. And after the observation of the evolution of that initial set we found the typical attracting set of the network. The network evolves according to the structure of the network and the Boolean function. The union of the attracting set that appears in the evolutionary result is a typical attracting set of the instance.

The experimental network size we choose 20,30,40,50 nodes, the network structure we choose to fix the out-degree of 2,3,4,5,6. For each set of parameters we use the average of the 100 experimental results as the final results.

According to the methods from complex network analysis, we investigate these indexes with different degree distributions. From the viewpoint of topology of the network, we calculated nodes importance value by different methods. In different cases, specific nodes will be immunized by the index order of nodes importance in networks.

\subsection{Results}

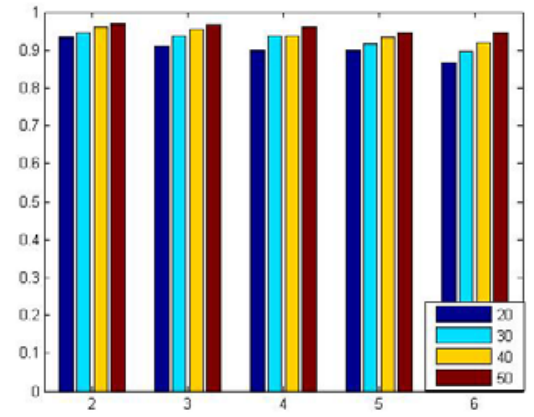

(a)Robustness (no immune)

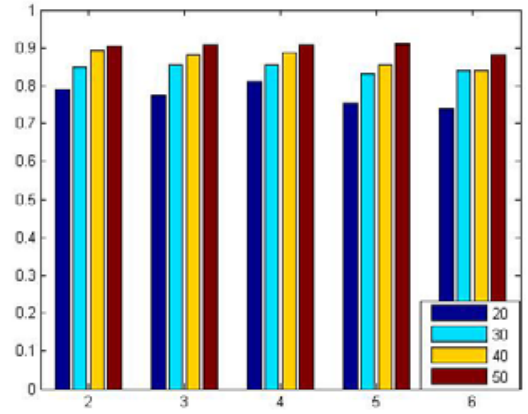

(b) Robustness (random immune (3 nodes)) 


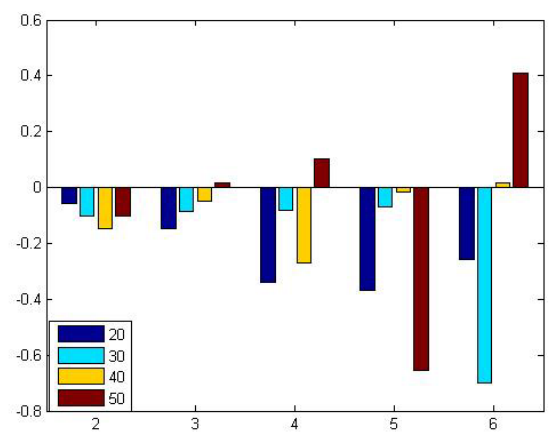

(c) Difference of $R \#$ (between random immune (3 nodes) and no immune)

Figure 1 Robustness and R\# of BA Networks (The abscissa represents the node's out-degree, and the different colors represent the number of different total nodes)

According to the results of Figure 1(a,b) and related experiments, the robustness of the boolean network is decreased after immunization. And average number of recovery is decreased too. Although immunization make state of Boolean network harder to recover to the same attracting set after disturbance, the recover speed is increased (recover to other attracting set also let the network stable).
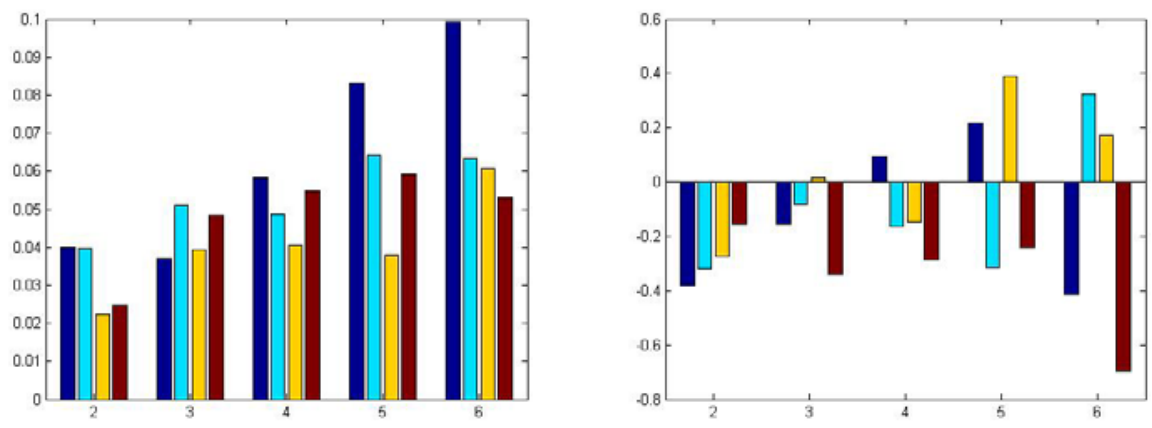

(a) R(robustness)

(b) R\#(average number of recovery steps)

Figure 2 Difference between immune by degree and random immune of BA Networks (5 nodes)

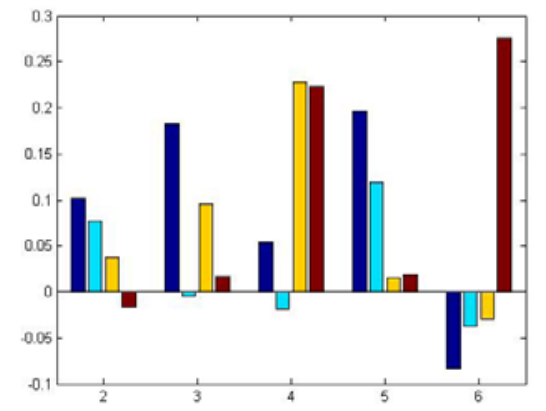

Figure 3 Difference of R\# between immune by degree and by local centrality of BA Networks (10 nodes)

And the robustness of immunization based on the degree index is higher than that of the random selection node. The average number of recovery steps based on the degree index is less than that of the random selection node. The average number of recovery steps of immunization based on local centrality is less then immunization by degree. So different immune strategy have different result. 
The immune operation of nodes in the Boolean network can be effective is the network becomes more stable, and targeted selection in the network structure of the important nodes can better control the Boolean network.

\section{Conclusions}

Based on the research of Boolean network and important node identification, the experiment of immune process on Boolean network is set up. Experiments show that the nodes in the immune network can effectively affect the stability of the network. And the important nodes in the immune network structure can obtain better results than the random selection nodes.

\section{Acknowledgements}

This work was supported by the Major Program of National Natural Science Foundation of China (11290141), National Natural Science Foundation of China (11401017, 11571028 and 11671025), Fundamental Research Funds for the Central Universities, Fundamental Research of Civil Aircraft No. MJ-F-2012-04, and Innovation Foundation of BUAA.

\section{References}

[1]Kauffman SA (1969) Metabolic Stability and Epigenesis in Randomly Constructed Genetic Nets. J Theor Biol 22: 437-467.

[2]Grefenstette J, Kim S, Kauffman S (2006) An analysis of the class of gene regulatory functions implied by a biochemical model. Bio Systems 84: 81-90.

[3]Shmulevich I, Kauffman SA (2004) Activities and Sensitivities in Boolean Network Models. Phys Rev Lett 93: 048701.

[4]Lloyd-Price J, Gupta A, Ribeiro A S. Robustness and information propagation in attractors of Random Boolean Networks[J]. PloS one, 2012, 7(7): e42018. MLA

[5]Barabási A L, Albert R, Jeong H. Scale-free characteristics of random networks: the topology of the worldwide web[J]. Physica A: statistical mechanics and its applications, 2000, 281(1): 69-77.

[6]Barabási A L, Albert R. Emergence of scaling in random networks[J]. science, 1999, 286(5439): 509-512.

[7]Lü L, Chen D, Ren X L, et al. Vital nodes identification in complex networks[J]. Physics Reports, 2016, 650: 1-63. 